\title{
On the Number of Enriques Quotients of a K3 Surface
}

\author{
By \\ Hisanori OHASHI*
}

\section{$\S 0 . \quad$ Introduction}

A $K 3$ surface $X$ is a compact complex surface with $K_{X} \sim 0$ and $H^{1}(X$, $\left.\mathcal{O}_{X}\right)=0$. An Enriques surface is a compact complex surface with $H^{1}\left(Y, \mathcal{O}_{Y}\right)=$ $H^{2}\left(Y, \mathcal{O}_{Y}\right)=0$ and $2 K_{Y} \sim 0$. The universal covering of an Enriques surface is a $K 3$ surface. Conversely every quotient of a $K 3$ surface by a free involution is an Enriques surface. Here a free involution is an automorphism of order 2 without any fixed points.

The moduli space of Enriques surfaces is constructed using the periods of their covering $K 3$ surfaces. Precisely speaking, an Enriques surface determines a lattice-polarized $K 3$ surface and vice versa, so that the moduli space of Enriques surfaces can be described by the moduli space of lattice-polarized $K 3$ surfaces. We note that even if we do not fix any polarization on Enriques surfaces, their covering $K 3$ surfaces automatically have a lattice-polarization. Then, what happens if we drop the lattice-polarization of the covering $K 3$ surface?

We will call two Enriques quotients of a $K 3$ surface distinct if they are not isomorphic to each other as varieties. In his paper [3], Kondo discovered a $K 3$ surface with two distinct Enriques quotients. He computed the automorphism groups of the two quotients. Since then, as far as the author knows, no other examples have been found.

In this paper we investigate this phenomenon. We show that $K 3$ surfaces with more than one distinct Enriques quotients have 9-dimensional components (neither irreducible nor closed) in the period domain. Moreover we compute the exact number of distinct Enriques quotients at a very general point of

Communicated by S. Mukai. Received April 11, 2006. Revised May 17, 2006.

2000 Mathematics Subject Classification(s): 14J28.

* Research Institute for Mathematical Sciences, Kyoto University, Kyoto 606-8502, Japan.

e-mail: pioggia@kurims.kyoto-u.ac.jp 
each component (Proposition 3.6). This generalizes Kondo's example in an arithmetic way and results in the following unboundedness theorem.

Theorem 0.1. For any nonnegative integer $l$, there exists a K3 surface $X$ with exactly $2^{l+10}$ distinct Enriques quotients. In particular, there does not exist a universal bound for the number of distinct Enriques quotients of a K3 surface.

We also generalize Kondo's example in a geometric way. Its construction is due to Mukai [4]. We introduce his construction and show that a generic Kummer surface $X$ of product type (see Section 4) has exactly 15 distinct Enriques quotients, which can be constructed from classical Lieberman's involution and Kondo-Mukai's involution.

Theorem 0.2. $\quad X$ has exactly 15 distinct Enriques quotients which are naturally in one-to-one correspondence with nonzero elements of the discriminant group of $N S(X)$.

From the theoretical point of view, we first show the following finiteness theorem on the automorphism group of a $K 3$ surface $X$ using a theorem of Borel.

Theorem 0.3. In $\operatorname{Aut}(X)$ there are only finitely many conjugacy classes of finite subgroups.

This theorem concerns us because it bounds the number of distinct Enriques quotients for any $K 3$ surfaces.

Corollary 0.4. $\quad$ Every $K 3$ surface $X$ has only finitely many distinct Enriques quotients.

The usage of the theorem of Borel is suggested by an anonimous referee. We remark that Corollary 0.4 follows also from our counting method described in Section 2. There, to count the exact number of distinct Enriques quotients, we consider more directly the embeddings of the Enriques lattice $U(2) \oplus E_{8}(2)$ into Néron-Severi lattices.

Notations and Convention. Our main tool is the theory of lattices and their discriminant forms. Here we collect some basic definitions about them. See [7] for the detailed exposition.

A lattice $L$ is a free $\mathbb{Z}$-module of finite rank equipped with a $\mathbb{Z}$-valued symmetric bilinear form. $L$ is said to be even if for all $l \in L, l^{2} \in 2 \mathbb{Z}$. In this 
paper we treat only even lattices, so that we sometimes omit mentioning the evenness. For a lattice $L$, there is a natural homomorphism $c: L \rightarrow L^{*}=$ $\operatorname{Hom}(L, \mathbb{Z})$ defined by $l \mapsto(l, \cdot)$. $L$ is said to be nondegenerate if $c$ is injective, and unimodular if $c$ is bijective. For $m \in \mathbb{Q}, L(m)$ denotes the same underlying group equipped with the form multiplied by $m$, assuming that it is $\mathbb{Z}$-valued. $U, E_{8}$ and $D_{4}$ denote the lattices given by the matrix $\left(\begin{array}{ll}0 & 1 \\ 1 & 0\end{array}\right)$, the Dynkin diagrams of type $E_{8}$ and $D_{4}$ respectively. We understand the latter two to be negative definite.

A finite quadratic form is a triple $(A, q, b)$ where $A$ is a finite abelian group, $q$ is a map $A \rightarrow \mathbb{Q} / 2 \mathbb{Z}$ and $b$ is a bilinear map $A \times A \rightarrow \mathbb{Q} / \mathbb{Z}$ which is symmetric and satisfies

$$
q(x+y)=q(x)+q(y)+2 b(x, y), x, y \in A .
$$

In the following we abbreviate $b(x, y)$ (resp. $q(x)$ ) to $x y$ (resp. $x^{2}$ ) and sometimes $(A, q, b)$ to $(A, q)$. We call $x^{2}$ the norm of $x$. As in the lattice case, we have a natural homomorphism $c: A \rightarrow A^{*}=\operatorname{Hom}(A, \mathbb{Q} / \mathbb{Z})$ defined by using $b$. $(A, q)$ is said to be nondegenerate if $c$ is bijective.

For an even nondegenerate lattice $L$, we can canonically associate a finite quadratic form $\left(A_{L}, q_{L}\right)$, called the discriminant quadratic form of $L$, by putting $A_{L}:=L^{*} / L$ and $q_{L}$ is the one naturally induced from the linear extension of the form on $L$ to $L^{*} \subset L \otimes \mathbb{Q}$. The discriminant group of $U(2)$ (resp. $D_{4}$ ) is denoted by $u(2)$ (resp. $v(2))$.

For a lattice $L, O(L)$ (resp. $O\left(q_{L}\right)$ ) denotes the integral orthogonal group of $L$ (resp. of $\left(A_{L}, q_{L}\right)$ ). We note that there is a natural homomorphism $\sigma_{L}: O(L) \rightarrow O\left(q_{L}\right) . L_{\mathbb{R}}\left(\right.$ resp. $\left.L_{\mathbb{C}}\right)$ is the scalar extension of $L$ to $\mathbb{R}$ (resp. $\mathbb{C}$ ).

The author is grateful to Professor Shigeru Mukai for many helpful discussions and suggestions. He indicated the example in Section 4. He is also grateful to Professors Shigefumi Mori and Noboru Nakayama for many valuable comments throughout the seminars.

\section{$\S 1$. Finiteness of Conjugacy Classes of Finite Subgroups}

First we collect some basic definitions about $K 3$ surfaces. Let $X$ be a $K 3$ surface. It is known that all $K 3$ surfaces are diffeomorphic. A $K 3$ lattice is a lattice isomorphic to $H^{2}(X, \mathbb{Z})=U^{\oplus 3} \oplus E_{8}^{\oplus 2}$. $\omega_{X}$ is the period of $X$, namely $\mathbb{C} \omega_{X}=H^{2,0}(X) . N S(X)=\omega_{X}^{\perp} \cap H^{2}(X, \mathbb{Z})$ is the Néron-Severi lattice of $X$. $T_{X}=(N S(X))^{\perp}$ is the transcendental lattice of $X$. 
We recall the structure of the integral automorphism group $O(N S)$ of $N S(X)$.

\section{Definition 1.1.}

1. The positive cone $\mathcal{C}_{X}$ is the connected component of $\left\{x \in N S(X) \otimes \mathbb{R} \mid x^{2}>\right.$ $0\}$ which contains an ample divisor.

2. The ample cone $\mathcal{A}_{X}$ is the subcone of $\mathcal{C}_{X}$ generated as a semigroup by ample divisors multiplied by positive real numbers.

\section{Definition 1.2.}

1. The Weyl group $W_{X}$ of $X$ is the subgroup of $O(N S)$ generated by automorphisms of the form $s_{l}: x \mapsto x+(x l) l$ for all elements $l \in N S(X)$ with $l^{2}=-2$.

2. $O^{\uparrow}(N S):=\left\{\varphi \in O(N S) \mid \varphi\left(\mathcal{C}_{X}\right)=\mathcal{C}_{X}\right\}$.

3. $O^{+}(N S):=\left\{\varphi \in O(N S) \mid \varphi\left(\mathcal{A}_{X}\right)=\mathcal{A}_{X}\right\}$.

4. $O_{0}(N S):=\operatorname{ker}\left(\sigma_{N S}: O(N S) \rightarrow O\left(q_{N S}\right)\right)$.

Further we use the abbreviations

$$
O_{0}^{\uparrow}(N S)=O_{0}(N S) \cap O^{\uparrow}(N S) \text { and } O_{0}^{+}(N S)=O_{0}(N S) \cap O^{+}(N S) .
$$

$O^{\uparrow}(N S)$ is of index 2 in $O(N S)$. The inclusions $O_{0}(N S) \subset O(N S)$ and $O_{0}^{+}(N S) \subset O^{+}(N S)$ are of finite index since $O\left(q_{N S}\right)$ is a finite group.

The following relation between these subgroups are important.

Proposition 1.3. We have (1) $W_{X} \subset O_{0}^{\uparrow}(N S),(2) O^{\uparrow}(N S)=W_{X} \rtimes$ $O^{+}(N S)$ and $(3) O_{0}^{\uparrow}(N S)=W_{X} \rtimes O_{0}^{+}(N S)$.

Proof. Since the generator $s_{l} \in W_{X}$ acts trivially on the hyperplane $H_{l}$ orthogonal to $l$ which intersects with the positive cone, $W_{X}$ preserves the positive cone. If $x \in N S^{*}$, then $x l \in \mathbb{Z}$ and $s_{l}(x)=x$ modulo $\mathbb{Z} l$. This proves (1). We have the semidirect product decomposition as in (2) because $W_{X}$ and $O^{\uparrow}(N S)$ are discrete subgroups of the isometry group of the Lobačevskii space modeled in $\mathcal{C}_{X}$ and $W_{X}$ is a reflection group with ample cone as its fundamental domain. See [12]. The proof of (3) is the same. 
We proceed to the proof of Theorem 0.3. For brevity, we say that a group $G$ has property $(F P)$ if $G$ has only finitely many conjugacy classes of finite subgroups. For example, let $G$ be an algebraic group defined over $\mathbb{Q}$. Then $G_{\mathbb{Z}}$ has property $(F P)$ by [11, Theorem 4.3] which we call the theorem of Borel.

\section{Lemma 1.4.}

(1) Let $\alpha: G \rightarrow K$ be a homomorphism of groups. If im $\alpha$ has property (FP) and $\operatorname{ker} \alpha$ is finite, then $G$ has property $(F P)$.

(2) Let $G=W \rtimes K$ be a semidirect decomposition of a group $G$. If two finite subgroups $F_{1}, F_{2} \subset K$ are $G$-conjugate, then they are also $K$-conjugate. In particular if $G$ has property $(F P)$, then so does $K$.

(3) Let $H \subset G$ be a subgroup of finite index. If $G$ has property $(F P)$, then so does $H$.

Proof. (1) Let $P_{1}, \cdots, P_{n}$ be the complete representatives of conjugacy classes of finite subgroups of im $\alpha$. Then any conjugacy class of finite subgroups of $G$ has a representative included in at least one of $\alpha^{-1}\left(P_{j}\right),(j=1, \cdots, n)$.

(2) Assume $F_{2}=g F_{1} g^{-1} . g$ can be written as $g=w k, w \in W, k \in K$. If $f_{i} \in F_{i}$ satisfy $f_{2}=g f_{1} g^{-1}$, then we have $\left(k f_{1} k^{-1}\right)^{-1} f_{2}=\left(k f_{1}^{-1} k^{-1} w k f_{1} k^{-1}\right) w^{-1} \in$ $W \cap K=\{1\}$. Hence $k f_{1} k^{-1}=f_{2}$. Thus $F_{1}$ and $F_{2}$ are conjugate by $k \in K$.

(3) Again let $P_{1}, \cdots, P_{n}$ be the complete representatives of conjugacy classes of finite subgroups of $G$. We put $G / H=\left\{a_{1} H, \cdots, a_{r} H\right\}$. Then the conjugacy classes of finite subgroups of $H$ are represented by $\left\{a_{i}^{-1} P_{j} a_{i} \mid i=1, \cdots, r\right.$ and $j=$ $1, \cdots, n\}$.

Now we show Theorem 0.3. In our words,

Theorem 1.5. Let $X$ be a K3 surface. Then $\operatorname{Aut}(X)$ has property $(F P)$.

Proof. First we assume that $X$ is projective. Consider the representation $r: \operatorname{Aut}(X) \rightarrow O(N S)$. Since every automorphism in ker $r$ fixes an ample divisor, $\operatorname{ker} r$ is finite. Thus it is enough to show that $\operatorname{im} r$ has the property (FP) by Lemma 1.4 (1).

By the theorem of Borel above, $O(N S)$ has property $(F P)$. Then, by Lemma 1.4 and Proposition 1.3, the property $(F P)$ goes down to $O^{\uparrow}(N S)$ and $O^{+}(N S)$. Now by the global Torelli theorem [10], im $r$ contains $O_{0}^{+}(N S)$, since $\varphi \in O_{0}^{+}(N S)$ preserves the ample cone $\mathcal{A}_{X}$ and can be extended to an isometry of $H^{2}(X, \mathbb{Z})$ which acts trivially on $T_{X}$. Thus we obtain

$$
O_{0}^{+}(N S) \subset \operatorname{im} r \subset O^{+}(N S)
$$


and these inclusions are of finite index. We see that im $r$ has the property $(F P)$ by Lemma $1.4(3)$.

Next we assume that $X$ is not projective. Nikulin [8] shows that any automorphism of $X$ of finite order acts on $T_{X}$ trivially. Therefore it is enough to show that $G=\operatorname{ker}\left(\operatorname{Aut}(X) \rightarrow O\left(T_{X}\right)\right)$ has property $(F P)$. We consider the representation $r: G \rightarrow O(N S)$.

If $\operatorname{alg} \cdot \operatorname{dim}(X)=0$, then $N S(X)$ is negative definite. Hence $N S(X) \oplus T_{X}$ is of finite index in $H^{2}(X, \mathbb{Z}), r$ is injective and $G$ is finite since $O(N S)$ is a finite group.

If $\operatorname{alg} \cdot \operatorname{dim}(X)=1$, then $N S(X)$ has one-dimensional kernel $\mathbb{Z} e$ and $Q:=$ $N S(X) / \mathbb{Z} e$ is negative definite. Every element of $G$ fixes $e$ since $e^{2}=0$ and exactly one of $e$ and $-e$ is represented by an effective cycle. Thus $r$ induces $s: G \rightarrow O(Q)$. Let $g \in G$ be an element of finite order. Since the fixed part $H^{2}(X, \mathbb{Z})^{g}$ is nondegenerate by the lemma below, it follows that if $s(g)=\operatorname{id}_{Q}$ then $g=\operatorname{id}_{X}$. On the other hand, $O(Q)$ is a finite group. Thus $\operatorname{Aut}(X)$ has only finitely many elements of finite order and $\operatorname{Aut}(X)$ has property $(F P)$.

Lemma 1.6. Let $L$ be a nondegenerate lattice and let $g$ be an isometry of $L$ of finite order $n$. Let $M=L^{g}=\{x \in L \mid g x=x\}$ be the fixed lattice. Then $M$ is nondegenerate.

Proof. Let $0 \neq x \in M$. Since $L$ is nondegenerate, there exists $y \in L$ with $x y \neq 0$. Put $z=y+g(y)+\cdots+g^{n-1}(y)$. Obviously $z \in M$ and we have

$$
x z=x y+g(x) g(y)+\cdots+g^{n-1}(x) g^{n-1}(y)=n x y \neq 0 .
$$

Therefore $M$ is nondegenerate.

As a corollary, Corollary 0.4 follows. In fact, if two free involutions $i_{1}$ and $i_{2}$ are conjugate by an automorphism $g$, then $g$ induces an isomorphism between $X / i_{1}$ and $X / i_{2}$.

\section{$\S 2 . \quad$ Number of Distinct Enriques Quotients}

The isomorphism classes of Enriques quotients are exactly the conjugacy classes of free involutions by the next proposition.

Proposition 2.1. $\quad$ Let $X$ be a $K 3$ surface and let $i_{1}$ and $i_{2}$ be free involutions on $X$. Then, $X / i_{1}$ and $X / i_{2}$ are isomorphic if and only if there exists an automorphism $g$ of $X$ such that $g i_{1} g^{-1}=i_{2}$. 
Proof. The "if" part is trivial; see the sentence after Lemma 1.6. Conversely, let $h$ be an isomorphism from $Y_{1}:=X / i_{1}$ to $Y_{2}:=X / i_{2}$. It induces an isomorphism of the canonical line bundles $h^{*} \mathcal{K}_{Y_{2}} \rightarrow \mathcal{K}_{Y_{1}}$. Since $X$ and $Y_{1}, Y_{2}$ are related as $X=\operatorname{Spec}\left(\mathcal{O}_{Y_{j}} \oplus \mathcal{K}_{Y_{j}}\right) j=1,2, h$ induces the following commutative diagram

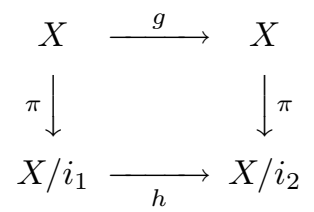

where $\pi$ denotes the covering map. It is clear that $g$ is the desired automorphism of $X$.

We put

$\mathfrak{M}:=$

$$
\left\{M \subset N S \mid \begin{array}{l}
\text { a primitive sublattice which satisfies } \\
(\mathrm{A}): M \cong U(2) \oplus E_{8}(2) \\
\text { (B) : No vector of square }-2 \text { in } N S(X) \text { is orthogonal to } M .
\end{array}\right\} .
$$

Recall that $U \oplus E_{8}$ is the Enriques lattice (modulo torsion) and $U(2) \oplus E_{8}(2)$ is the pullback in the covering $K 3$ lattice. For each $M \in \mathfrak{M}$, we define an isometry $i_{M}: H^{2}(X, \mathbb{Z}) \rightarrow H^{2}(X, \mathbb{Z})$ by $i_{M}(m)=m$ when $m \in M$ and $i_{M}(n)=$ $-n$ when $n$ is orthogonal to $M$. This is well-defined because $M \cong U(2) \oplus E_{8}(2)$ is 2-elementary.

Proposition 2.2. On a K3 surface $X$, there is a one-to-one correspondence between free involutions on $X$ and primitive sublattices $M$ of $N S(X)$ which satisfy (A) and (B) above and the following

(C) $M$ contains an ample divisor.

In other words, $i_{M}$ defined above is a free involution if and only if $M$ contains an ample divisor. Also any free involution can be written in the form $i_{M}$.

Proof. We associate a free involution with its invariant sublattice in $H^{2}(X, \mathbb{Z})$. The statement follows from [6, Corollary 2.5], [9, Theorem 4.2.2, p. 1426] and the strong Torelli theorem for $K 3$ surfaces [10]. In [9], the assumption is slightly different from ours, but the same proof goes.

To count the number of distinct Enriques quotients, we consider the natural action of $O(N S)$ on $\mathfrak{M}$,

$$
O(N S) \ni \varphi: M \mapsto \varphi(M) \in \mathfrak{M} .
$$


Corresponding lattice automorphisms satisfy $i_{\varphi(M)}=\varphi i_{M} \varphi^{-1}$.

In the following, $\operatorname{Aut}\left(T_{X}, \omega_{X}\right)$ is the subgroup of $O\left(T_{X}\right)$ consisting of the integral orthogonal transformations whose scalar extention to $\mathbb{C}$ preserves the period $\mathbb{C} \omega_{X} \subset T_{X} \otimes \mathbb{C}$.

Theorem 2.3. Let $M_{1}, \cdots, M_{k} \in \mathfrak{M}$ be a (finite) complete set of representatives for the action of $O(N S)$ on $\mathfrak{M}$. For each $j=1, \cdots, k$, let

$$
K^{(j)}=\left\{\varphi \in O(N S) \mid \varphi\left(M_{j}\right)=M_{j}\right\}
$$

be the stabilizer subgroup of $M_{j}$ and $\sigma\left(K^{(j)}\right)$ its canonical image in $O\left(q_{N S}\right)$. We put

$$
B_{0}=\sum_{j=1}^{k} \#\left(O\left(q_{N S}\right) / \sigma\left(K^{(j)}\right)\right) .
$$

(1) The number of distinct Enriques quotients of $X$ does not exceed $B_{0}$.

(2) If $\sigma: O(N S) \rightarrow O\left(q_{N S}\right)$ is surjective and if $\operatorname{Aut}\left(T_{X}, \omega_{X}\right)=\{ \pm \mathrm{id}\}$, then $X$ has exactly $B_{0}$ distinct Enriques quotients.

Proof. First we remark that by Proposition 1.15.1 in [7], the set of representatives is always finite. In view of Proposition 2.1, we can count the number of distinct Enriques quotients separately for each orbit $O(N S) \cdot M_{j}$. Hence, for simplicity, we fix an orbit and omit the index $j$ so that we use the symbols $M:=M_{j}, \mathcal{O}:=O(N S) \cdot M$ and $K=K^{(j)}$.

Step 1. $\mathcal{O}$ contains an element which corresponds to a free involution.

Proof. The following is a standard argument used in [10]. Our proof is taken from [6]. By the condition (A) in Proposition 2.2, $M \cap \mathcal{C}_{X} \neq \emptyset$. Consider in $\mathcal{C}_{X}$ countably many hyperplanes $H_{d}=\left\{x \in N S_{\mathbb{R}} \mid x d=0\right\}$, where $d$ runs over $(-2)$ vectors in $N S$. The union $\cup H_{d}$ is a locally finite closed subset in $\mathcal{C}_{X}$ and does not contain $M$ by the condition (B). The complement $\mathcal{C}_{X}-\cup H_{d}$ is a collection of (at most) countably many connected open sets, namely chambers, which corresponds to the elements of $W_{X}$ in one-to-one way. The ample cone $\mathcal{A}_{X}$ equals one of the chambers. Thus if we choose $v \in M \cap \mathcal{C}_{X}-\cup H_{d}$, there exists $\varphi \in W_{X} \subset O(N S)$ such that $\varphi(v)$ is an ample divisor.

Thus we can assume that $i_{M}$ is already a free involution of $X$. Next we set

$$
\mathfrak{N}:=\left\{M^{\prime} \in \mathcal{O} \mid i_{M^{\prime}} \text { is a free involution }\right\} .
$$

Step 2. $\mathfrak{N}=O^{+}(N S) \cdot M$. 
Proof. $\supset$ follows from Proposition 2.2. Let $\varphi \in O(N S)$ and suppose $i_{\varphi(M)}$ is a free involution. We can assume $\varphi \in O^{\uparrow}(N S)$, since otherwise $-\varphi \in$ $O^{\uparrow}(N S)$ and $(-\varphi)(M)=\varphi(M)$. By Proposition 1.3, we can write $\varphi=w \psi$ with $w \in W_{X}$ and $\psi \in O^{+}(N S)$. Using Lemma $1.4(2), i_{\varphi(M)}=w i_{\psi(M)} w^{-1}$ implies $i_{\varphi(M)}=i_{\psi(M)}$. Therefore $\varphi(M)=\psi(M)$.

Step 3. Let $\psi_{1}, \psi_{2} \in O^{+}(N S)$. If $\sigma\left(\psi_{j}\right)$ have the same class in $O\left(q_{N S}\right) /$ $\sigma(K)$, then $i_{\psi_{1}(M)}$ and $i_{\psi_{2}(M)}$ are conjugate in $\operatorname{Aut}(X)$.

Proof. By the assumption $\sigma\left(\psi_{1}^{-1} \psi_{2}\right) \in \sigma(K)$, so there exists $\varphi \in K$ such that $\sigma(\varphi)=\sigma\left(\psi_{1}^{-1} \psi_{2}\right)$. It follows that $\sigma\left(\psi_{1} \varphi \psi_{2}^{-1}\right)=\mathrm{id}$, so that $\left.\psi_{1} \varphi \psi_{2}^{-1}\right|_{N S}$ together with $\mathrm{id}_{T_{X}}$ gives an automorphism $a$ of $X$, by the Torelli theorem. It follows that

$$
a i_{\psi_{2}(M)} a^{-1}=i_{a \psi_{2}(M)}=i_{\psi_{1} \varphi(M)}=i_{\psi_{1}(M)} .
$$

By now, we have proved that $\mathcal{O}$ contains at most $\# O\left(q_{N S}\right) / \sigma(K)$ distinct Enriques quotients. Assertion (1) follows.

Step 4. If $\operatorname{Aut}\left(T_{X}, \omega_{X}\right)=\{ \pm \mathrm{id}\}$, then the converse of Step 3 holds.

Proof. Assume there exists $\varphi \in \operatorname{Aut}(X)$ such that $\varphi i_{\psi_{1}(M)} \varphi^{-1}=i_{\psi_{2}(M)}$, which is equivalent to $\varphi \psi_{1}(M)=\psi_{2}(M)$ and to $\psi_{2}^{-1} \varphi \psi_{1} \in K$. By the assumption, $\sigma(\varphi)= \pm$ id which is contained in the center of $O\left(q_{N S}\right)$. It follows that $\pm \sigma\left(\psi_{2}^{-1} \psi_{1}\right) \in \sigma(K)$. We remark that $-\mathrm{id} \in \sigma(K)$ since $\sigma\left(i_{M}\right)=-\mathrm{id}$. Therefore we get $\sigma\left(\psi_{2}^{-1} \psi_{1}\right) \in \sigma(K)$.

Step 5. If $\sigma$ is surjective, then the restriction $\left.\sigma\right|_{O^{+}(N S)}$ is also surjective.

Proof. Put $N=M^{\perp}$ in $N S$. Since $M$ is 2-elementary, $t=\left(-\operatorname{id}_{M}, \operatorname{id}_{N}\right)$ extends to an isometry of $N S$. $t$ doesn't preserve the positive cone. Therefore $O(N S)$ is generated by $t$ and $O^{\uparrow}(N S)$. This implies the surjectivity of $O^{\uparrow}(N S) \rightarrow O\left(q_{N S}\right)$. By Proposition 1.3, the assertion follows.

Now the proof is complete.

Lastly we mention a useful theorem of Nikulin in [7] which saves us from checking one of the conditions in Theorem $2.3(2)$.

Theorem 2.4 (Nikulin). $\quad$ Let $T$ be an even indefinite nondegenerate lattice satisfying the following two conditions:

(1) $\operatorname{rank}(T) \geq l\left(A_{T_{p}}\right)+2$ for all prime numbers $p$ except for 2 .

(2) if $\operatorname{rank}(T)=l\left(A_{T_{2}}\right)$, then $q_{T_{2}}$ contains a component $u(2)$ or $v(2)$.

Then the genus of $T$ contains only one class, and the homomorphism $O(T) \rightarrow O\left(q_{T}\right)$ is surjective. Here $A_{T_{p}}$ denotes the $p$-component of the finite abelian group $A_{T}$ and $l$ denotes the number of minimal generators. 


\section{$\S 3 . \quad$ Enriques Quotients of $K 3$ Surfaces in the Heegner Divisors}

In this section we prove Theorem 0.1. We deal with certain divisors of the period domain $\mathfrak{D}$ of $U(2) \oplus E_{8}(2)$-polarized marked $K 3$ surfaces. Fix the unique primitive embedding of $U(2) \oplus E_{8}(2)$ in the $K 3$ lattice $\Lambda$. Then $\mathfrak{D}$ is by definition

$$
\mathfrak{D}:=\left\{[\omega] \in \mathbb{P}\left(\left(U(2) \oplus E_{8}(2)\right) \frac{\perp}{\mathbb{C}}\right) \mid \omega^{2}=0, \omega \bar{\omega}>0\right\} .
$$

Here $\mathbb{P}(V)$ means the associated projective space of a complex vector space $V$, which consists of all lines through the origin. It follows from the surjectivity of the period map that every point of $\mathfrak{D}$ corresponds to a $K 3$ surface $X$ with a marking $H^{2}(X, \mathbb{Z}) \cong \Lambda$.

Let $S \subset \Lambda$ be a primitive sublattice of rank 11 containing the lattice $U(2) \oplus E_{8}(2)$ fixed above. Then the subset

$$
\mathfrak{D}(S):=\left\{[\omega] \in \mathbb{P}\left(S_{\mathbb{C}}^{\perp}\right) \mid \omega^{2}=0, \omega \bar{\omega}>0\right\}
$$

is called the Heegner divisor of type $S$ in $\mathfrak{D}$. Let $X$ be a marked $K 3$ surface whose period is in $\mathfrak{D}(S)$. Since $N S(X)$ is written as $\Lambda \cap \omega_{X}^{\perp}, N S(X)$ contains the primitive sublattice $S$.

Proposition 3.1. If $X$ corresponds to a very general point of $\mathfrak{D}(S)$, namely to a point in the complement of a union of countably many closed analytic subset of $\mathfrak{D}(S)$, then we have $N S(X)=S$ and $\operatorname{Aut}\left(T_{X}, \omega_{X}\right)=\{ \pm \operatorname{id}\}$.

Proof. This is a well-known fact. For the latter, the same proof as in [1, Lemma 2.9] works.

We consider the case when

$$
S=U(2) \oplus E_{8}(2) \oplus\langle-2 N\rangle,
$$

where $\langle-2 N\rangle$ is the rank 1 lattice whose generator $g$ has $g^{2}=-2 N$. It is easy to see that the $K 3$ lattice $\Lambda$ contains $S$ as a primitive sublattice. We fix it once and for all. The discriminant form of $S$ is isomorphic to $q=u(2)^{\oplus 5} \oplus c(-2 N)$, where $c(-2 N)$ is the discriminant form of $\langle-2 N\rangle$.

Let the integer $N$ be $N=4 p_{1} \cdots p_{l}$, where $p_{1}, \cdots, p_{l}$ are distinct odd prime numbers. In the next we compute the order of $O(q)$.

\section{Lemma 3.2.}

$$
\begin{aligned}
& \#\left\{x \in c(-2 N) \mid \operatorname{ord}(x)=2 N, x^{2} \equiv-1 / 2 N(\bmod 2 \mathbb{Z})\right\}=2^{l+1}, \\
& \#\left\{x \in c(-2 N) \mid \operatorname{ord}(x)=2 N, x^{2} \equiv 1-1 / 2 N(\bmod 2 \mathbb{Z})\right\}=2^{l+1} .
\end{aligned}
$$


Proof. The left-hand-side of the first equality is

$$
\begin{aligned}
\# & \left\{k \in \mathbb{Z} \mid(k, 2 N)=1,1 \leq k \leq 2 N-1 \text { and }-k^{2} / 2 N=-1 / 2 N \in \mathbb{Q} / 2 \mathbb{Z}\right\} \\
& =(1 / 2) \#\left\{k \in \mathbb{Z} \mid(k, 4 N)=1,1 \leq k \leq 4 N-1 \text { and } k^{2}-1 \equiv 0(\bmod 4 N)\right\} \\
& =(1 / 2) \#\left\{x \in(\mathbb{Z} / 4 N \mathbb{Z})^{\times} \mid \operatorname{ord}(x)=1 \text { or } 2\right\} .
\end{aligned}
$$

Then we can use the structure theorem of the unit group $(\mathbb{Z} / 4 N \mathbb{Z})^{\times}$. Similarly the left-hand-side of the latter is

$$
\begin{aligned}
\# & \left\{k \in \mathbb{Z} \mid(k, 2 N)=1,1 \leq k \leq 2 N-1 \text { and }-k^{2} / 2 N=1-1 / 2 N \in \mathbb{Q} / 2 \mathbb{Z}\right\} \\
& =(1 / 2) \#\left\{k \in \mathbb{Z} \mid(k, 4 N)=1,1 \leq k \leq 4 N-1 \text { and } k^{2} \equiv 1-2 N(\bmod 4 N)\right\} \\
& =(1 / 2) \#\left\{k \in \mathbb{Z} \mid(k, 4 N)=1,1 \leq k \leq 4 N-1, k^{2}\right. \\
& \left.\equiv 1(\bmod 2 N) \text { and } k^{2} \not \equiv 1(\bmod 4 N)\right\} .
\end{aligned}
$$

Using the commutative diagram

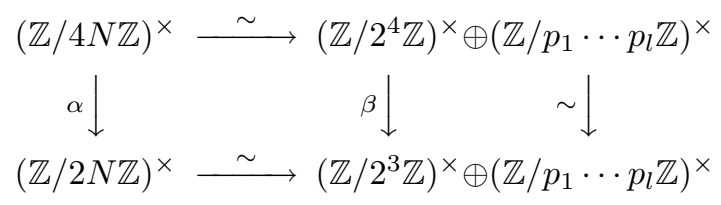

where $\alpha, \beta$ are both 2:1 maps, we can count the number of elements which have order 2 in the bottom row but do not in the top row.

Proposition 3.3. $O(q)$ acts transitively on the set of elements $x \in q$ with $x^{2} \equiv-1 / 2 N(\bmod 2 \mathbb{Z})$. There are $2^{11+l}$ such elements.

Proof. Such element $x$ generates a subgroup $\langle x\rangle$ isomorphic to $c(-2 N)$. Since it is nondegenerate, $\langle x\rangle$ is a direct summand in $q$. This implies the transitivity. If we put the number of elements in $u(2)^{\oplus 5}$ with norm 0 to be $A$ and norm $12^{10}-A$, we can compute the length of the orbit as $2^{l+1} \cdot A+2^{l+1}$. $\left(2^{10}-A\right)=2^{11+l}$.

Under these computations we can prove

Theorem 3.4. $\quad$ For any nonnegative integer $l$, there exists a K3 surface $X$ with exactly $2^{l+10}$ distinct Enriques quotients.

Proof. By Proposition 3.1, there exists a $K 3$ surface $X$ such that $N S(X) \cong$ $S$ and $\operatorname{Aut}\left(T_{X}, \omega_{X}\right)=\{ \pm \mathrm{id}\}$. We show that the primitive embedding of 
$U(2) \oplus E_{8}(2)$ into $N S(X)$ is unique under the action of $O(N S)$. In fact, since $N S(1 / 2)$ is again an even lattice, we have a natural identification

$$
\operatorname{Hom}\left(U(2) \oplus E_{8}(2), N S\right)=\operatorname{Hom}\left(U \oplus E_{8}, N S(1 / 2)\right) .
$$

We see that any primitive embedding as above is a direct summand. This clearly implies the uniqueness.

Obviously $N S(X)$ has a primitive sublattice $M$ isomorphic to $U(2) \oplus E_{8}(2)$ and $M^{\perp}=\langle-2 N\rangle$. Let $K$ be the stabilizer group of $M$ and $\sigma(K)$ its canonical image in $O\left(q_{N S}\right)$. Since $N S \cong M \oplus M^{\perp}$ we see that $K=O(M) \times O\left(M^{\perp}\right)=$ $O(M) \times\left\{ \pm \operatorname{id}_{M^{\perp}}\right\}$. On the other hand by Theorem $2.4 \sigma_{M}: O(M) \rightarrow O\left(q_{M}\right)$ is surjective. This shows $\sigma(K)=O\left(u(2)^{\oplus 5}\right) \times\{ \pm \mathrm{id}\} \subset O\left(q_{M} \oplus q_{M^{\perp}}\right)=O\left(q_{N S}\right)$. Thus $\#\left(O\left(q_{N S}\right) / \sigma(K)\right)=\# O\left(q_{N S}\right) / 2 \# O\left(u(2)^{\oplus 5}\right)=2^{10+l}$ by Proposition 3.3. This together with Theorems 2.3 and 2.4 completes the proof.

In fact we can classify all the possible Néron-Severi lattices of a $K 3$ surface with Picard number 11 having an Enriques quotient. In each case, we can compute the number of Enriques quotients as follows by an explicit calculation. Details are omitted. The result is as follows.

Proposition 3.5. $\quad$ Let $X$ be a K3 surface with Picard number 11 having an Enriques quotient. Then the Néron-Severi lattice of $X$ is one of the followings.

$$
\begin{array}{lc}
\text { Type I : } U(2) \oplus E_{8}(2) \oplus\langle-2 N\rangle & (N \geq 2) \\
\text { Type II : } U \oplus E_{8}(2) \oplus\langle-4 M\rangle & (M \geq 1) .
\end{array}
$$

If we put $2 N=2^{e} p_{1}^{e_{1}} \cdots p_{l}^{e_{l}}$ in type I, or $4 M=2^{e} p_{1}^{e_{1}} \cdots p_{l}^{e_{l}}$ in type II, the bound $B_{0}$ in Theorem 2.3 is as follows.

\section{Proposition 3.6.}

$$
B_{0}= \begin{cases}2^{l-1} & \text { in Type I and } e=1 \\ \left(2^{5}+1\right) \cdot 2^{l+4} & \text { in Type I and } e=2 \\ 2^{l+10} & \text { in Type I and } e \geq 3 \\ 1 & \text { in Type II and } e=2, \quad l=0 \\ 2^{l-1} & \text { in Type II and } e=2, \quad l>0 \\ 2^{2 l+5} & \text { in Type II and } e \geq 3\end{cases}
$$

The lattice $S$ we used fits in the third case. 


\section{$\S 4$. Enriques Quotients of Generic Kummer Surfaces of Product Type}

Kondo found the first example of a $K 3$ surface which has two distinct Enriques quotients in [3, Remark 3.5.3], where he computed the automorphism groups of the two quotients. Recently Mukai generalized Kondo's example which we now describe.

Kummer surfaces of product type. Let $C_{1}$ and $C_{2}$ be elliptic curves and construct the Kummer surface as $X=K m\left(C_{1} \times C_{2}\right)$. We put the 2-torsion points of $C_{1}$ (resp. $C_{2}$ ) as $\left\{b_{1}=0, b_{2}, b_{3}, b_{4}\right\}$ (resp. $\left\{c_{1}=0, c_{2}, c_{3}, c_{4}\right\}$ ) and denote by $\delta$ the natural rational map of $C_{1} \times C_{2}$ to $X$. Let $E_{k}$ (resp. $F_{k}$ ) be the image of $C_{1} \times\left\{c_{k}\right\}$ (resp. $\left\{b_{k}\right\} \times C_{2}$ ) by $\delta$. Then $X$ has the configuration of 24 smooth rational curves as in Figure 1, where $G_{i j}$ is the exceptional curve corresponding to $\left(b_{i}, c_{j}\right) \in C_{1} \times C_{2}$.

Sometimes it is called the double Kummer configuration. In the following we introduce two kinds of free involutions on $X$ with parameters.

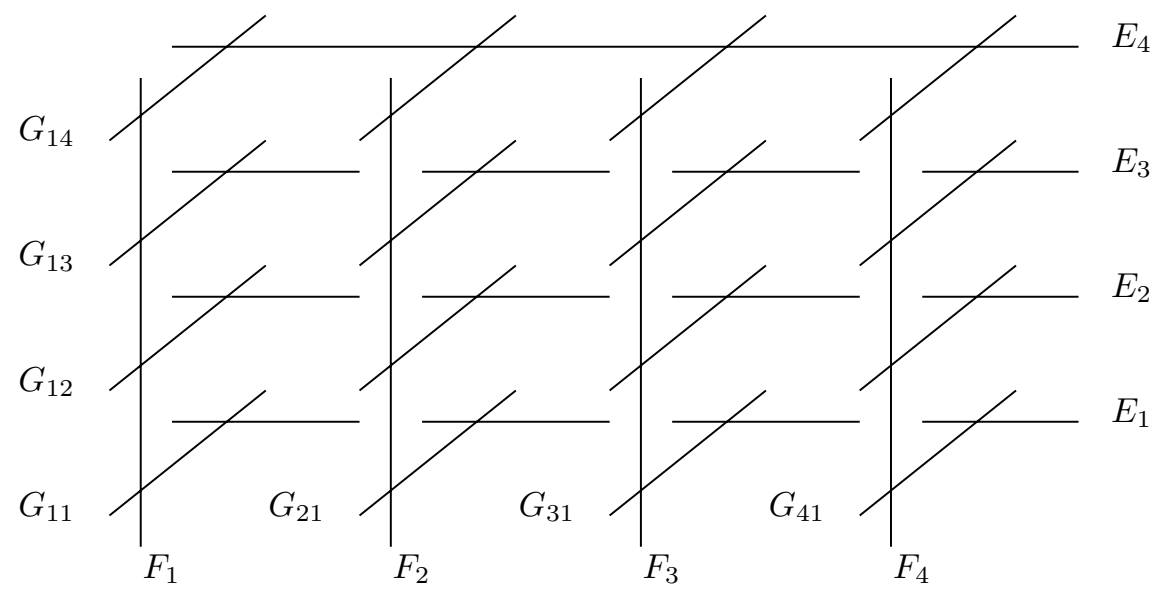

Figure 1. The double Kummer configuration

Involutions of Lieberman type. Let $a=\left(b_{i}, c_{j}\right) \in C_{1} \times C_{2}$ be a 2torsion point not lying on $C_{1} \times\{0\}$ or $\{0\} \times C_{2}$. Let $\tau$ (resp. $\rho$ ) be the involution of $X$ induced by the automorphism $\left(-\mathrm{id}_{C_{1}}, \operatorname{id}_{C_{2}}\right.$ ) (resp. the translation by $a$ ) of $C_{1} \times C_{2}$. Then $\sigma=\tau \rho$ is a free involution. We remark that $a$ has the parameter $i, j$ with $2 \leq i, j \leq 4$. 
Involutions of Kondo-Mukai type. Let $\tau$ be as above. $X / \tau$ is isomorphic to $\mathbb{P}^{1} \times \mathbb{P}^{1}$ with sixteen points blown up, which correspond to $G_{i j}$ 's in Figure 1. We regard $\mathbb{P}^{1} \times \mathbb{P}^{1}$ as a smooth quadric in $\mathbb{P}^{3}$ so that there are morphisms

$$
X \longrightarrow X / \tau \longrightarrow \mathbb{P}^{1} \times \mathbb{P}^{1} \subset \mathbb{P}^{3} .
$$

Choose two permutations $I=\left\{i_{1}, i_{2}, i_{3}, i_{4}\right\}$ and $J=\left\{j_{1}, j_{2}, j_{3}, j_{4}\right\}$ of $\{1,2$, $3,4\}$ and we put $g_{k}=\varepsilon\left(G_{i_{k} j_{k}}\right) \in \mathbb{P}^{1} \times \mathbb{P}^{1}$. We project $\mathbb{P}^{1} \times \mathbb{P}^{1}$ onto a hyperplane $\cong \mathbb{P}^{2}$ of $\mathbb{P}^{3}$ from $g_{4}$. This birational map $\varepsilon^{\prime}$ contracts two curves whose images we denote by $P$ and $Q \in \mathbb{P}^{2}$. Let $\bar{g}_{k}=\varepsilon^{\prime}\left(g_{k}\right), k=1,2,3$. Then we obtain two involutions on $X$ : One is the covering transformation $\nu$ of the degree 2 map $X \rightarrow \mathbb{P}^{2}$. The other involution $\mu$ is induced by the unique Cremona transformation of $\mathbb{P}^{2}$ centered at $\bar{g}_{1}, \bar{g}_{2}, \bar{g}_{3}$ interchanging $P$ and $Q$. Then $\sigma=\nu \mu$ is a free involution. It can be shown that $\sigma$ depends only on the set $\left\{G_{i_{k} j_{k}}\right\}_{1 \leq k \leq 4}$. See [4] for such canonical description of this involution. Therefore the parameter is the set $\left\{G_{i_{k} j_{k}}\right\}_{1 \leq k \leq 4}$. There are twenty-four choices of parameters in appearance, but we will reveal that there are essentially six.

If $C_{1}$ and $C_{2}$ are chosen to be general enough, we see that

$$
\begin{gathered}
N S(X) \cong U \oplus E_{8} \oplus D_{4}^{\oplus 2}, T_{X} \cong U(2)^{\oplus 2}, A_{N S(X)}=u(2)^{\oplus 2} \\
\text { and } \operatorname{Aut}\left(T_{X}, \omega_{X}\right)=\{ \pm \mathrm{id}\} .
\end{gathered}
$$

We will call such $X$ a generic Kummer surface of product type. In the following main theorem of this section we classify the all free involutions on $X$.

Theorem 4.1. $\quad$ Let $X$ be a generic Kummer surface of product type. Then $X$ has exactly fifteen distinct Enriques quotients which are naturally in one-to-one correspondence with nonzero elements of $A_{N S(X)}$. Moreover all of them can be geometrically constructed from the preceding examples by choosing appropriate parameters.

We remark that the Lieberman involutions correspond to nine elements of norm 0 of $A_{N S(X)}$ and Kondo-Mukai involutions to six elements of norm 1. In the rest of this section we prove Theorem 4.1. First we determine the isomorphism classes of primitive embeddings of $M:=U(2) \oplus E_{8}(2) \subset$ $S=U \oplus E_{8} \oplus D_{4}^{\oplus 2}$. This step is purely lattice-theoretic. We use the following theorem of Nikulin [7, Propositions 1.5.1 and 1.15.1].

Theorem 4.2. Let $M \subset S$ be a primitive embedding of even nondegenerate lattices. Put $N:=M^{\perp}$. Then the following isomorphisms exist. (Note 
that (1) expresses $A_{S}$ in terms of $A_{M}$ and $A_{N}$ while (2) expresses $A_{N}$ in terms of $A_{M}$ and $A_{S}$.)

(1) There are subgroups $\Gamma_{M} \subset A_{M}, \Gamma_{N} \subset A_{N}$ and a sign-reversing isometry $\gamma: \Gamma_{M} \rightarrow \Gamma_{N}$ such that if $\Gamma$ is the pushout of $\gamma$, namely $\Gamma=\{(x, \gamma(x)) \in$ $\left.A_{M} \oplus A_{N} \mid x \in \Gamma_{M}\right\}$, then

$$
q_{S} \cong\left(\left.q_{M} \oplus q_{N}\right|_{\Gamma^{\perp}} / \Gamma\right)
$$

(2) There are subgroups $\Gamma_{M} \subset A_{M}, \Gamma_{S} \subset A_{S}$ and a sign-reversing isometry $\gamma: \Gamma_{M} \rightarrow \Gamma_{S}$ such that if $\Gamma$ is the pushout of $\gamma$, then

$$
q_{N} \cong\left(-\left.q_{M} \oplus q_{S}\right|_{\Gamma^{\perp}} / \Gamma\right)
$$

Proposition 4.3. Let

$$
S=U \oplus E_{8} \oplus D_{4}^{\oplus 2} \text { and } M=U(2) \oplus E_{8}(2) .
$$

Assume $M$ is a primitive sublattice of $S$ which is orthogonal to no $(-2)$ vectors of $S$. Then

(1) $N:=M^{\perp}$ is isomorphic to $E_{8}(2)$.

(2) There are exactly two such primitive sublattices up to the action of $O(S)$.

Proof. By Theorem $4.2(2), A_{N}$ is a 2-elementary abelian group and $q_{N}$ takes only integral values on $A_{N}$. On the other hand, $N$ is a negative definite lattice of rank 8 . This implies

$$
A_{N} \cong \begin{cases}u(2)^{\oplus a}, & 0 \leq a \leq 4, \text { or } \\ v(2) \oplus u(2)^{\oplus a}, & 0 \leq a \leq 3 .\end{cases}
$$

by the decomposition theorem of 2-elementary finite quadratic forms (see [7, Proposition 1.8.1]).

Checking the signature of $\left(A_{N}, q_{N}\right)$, we see that the latter does not occur. In all other cases, we find that $N$ has a unimodular overlattice of rank 8 , i.e., $E_{8}$. The index $\left[E_{8}: N\right]$ is given by $2^{a}$.

Claim. Let $N \subset E_{8}$ be an overlattice and assume $N$ contains no (-2) vectors. Then $\left[E_{8}: N\right] \geq 9$.

Proof. We take a basis of $E_{8}$ as in Figure 2. Consider the elements $f_{0}:=0, f_{j}:=e_{1}+\cdots+e_{j}, 1 \leq j \leq 7$ and $f_{8}:=2 e_{1}+3 e_{2}+4 e_{3}+5 e_{4}+6 e_{5}+$ $4 e_{6}+2 e_{7}+3 e_{8}$. It is easy to see that any difference $f_{j}-f_{i}, 0 \leq i<j \leq 8$ has 


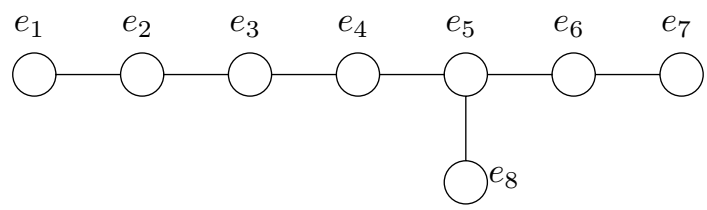

Figure 2. Dynkin diagram of type $E_{8}$

norm -2 . This means that every $f_{i}$ is a distinct element of the reminder class group $E_{8} / N$.

Thus we obtain $A_{N}=u(2)^{\oplus 4}$. Therefore $N$ has the maximal number of minimal generators of the discriminant group. This implies that for any $n \in N$, $n / 2 \in N^{*}$. In particular $N(1 / 2)$ is a unimodular lattice. Thus we see that $N \cong E_{8}(2)$ and (1) is proved.

For (2), we use the notation introduced in Theorem 4.2 (1). Since $q_{M} \oplus q_{N}$ is nondegenerate $\# \Gamma=\# \Gamma_{N}=\left(\# A_{M} \cdot \# A_{N} / \# A_{S}\right)^{1 / 2}=2^{7}$. Therefore $\# \Gamma_{N}^{\perp}=$ $\# A_{N} / \# \Gamma=2$ and we can put $\Gamma_{N}^{\perp}=\left\{0, z_{N}\right\}$. There are two cases where $q_{N}\left(z_{N}\right)=0,1$. Thus there are at least two distinct primitive embedding of $M \subset S$. On the other hand, the canonical homomorphism $\sigma_{M}: O(M) \rightarrow$ $O\left(q_{M}\right)$ and $\sigma_{N}: O(N) \rightarrow O\left(q_{N}\right)$ are both surjective by Proposition 2.4 and [1]. Thus the primitive embedding of $M \subset S$ is classified by the invariant $q_{N}\left(z_{N}\right)$ as we see from the following argument.

Claim. Let $M_{1}$ and $M_{2}$ be two primitive sublattices of $S$ satisfying the assumption. For each $k=1,2$, we use the same notation as above, indexed by $k$. If $q_{N_{1}}\left(z_{N_{1}}\right)=q_{N_{2}}\left(z_{N_{2}}\right)$, then there exists $\varphi \in O(S)$ which transforms $M_{1}$ onto $M_{2}$.

Proof. By Witt's theorem on the finite quadratic forms, there exist $\psi_{M}$ and $\psi_{N}$ fitting in the square inside.

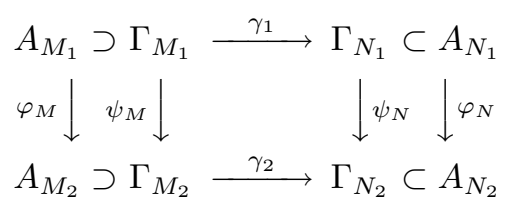

Again by Witt's theorem we can extend $\psi_{M}\left(\right.$ resp. $\left.\psi_{N}\right)$ to $\varphi_{M}\left(\right.$ resp. $\left.\varphi_{N}\right)$ in the diagram. By the surjectivity of $\sigma_{M}$ and $\sigma_{N}$ mentioned above, these isomorphisms lift to an isomorphism between $M_{1} \oplus N_{1}$ and $M_{2} \oplus N_{2}$ which preserves the overlattice $S$. This was the assertion. 
Remark 4.4. The same but geometric situation of the proposition is considered in [5]. There (1) is proved by a geometric method.

Now we regard $S$ as the Néron-Severi lattice $N S$ of a generic Kummer surface of product type. To see the natural correspondence in Theorem 4.1, we have to associate a free involution with an element of $A_{N S(X)}=A_{N S}$.

Definition 4.5. Let $M \cong U(2) \oplus E_{8}(2) \subset N S$ be a primitive sublattice. We use the same notation as in Proposition 4.3. Then we define the patching element $v_{M} \in A_{N S}$ associated with the primitive sublattice $M$ to be the image of the element $\left(0, z_{N}\right) \in A_{M} \oplus A_{N}$ in $A_{N S}$ under the isomorphism of Theorem 4.2 (1).

We note that $v_{M} \neq 0$. Also we note the equality $q_{N}\left(z_{N}\right)=q_{N S}\left(v_{M}\right)$. Using $v_{M}$, we can describe the group $\sigma_{N S}(K)$ in the Theorem 2.3. Recall that $K \subset O(N S)$ is the stabilizer subgroup of $M$.

Lemma 4.6. $\quad \sigma_{N S}(K)$ is equal to the stabilizer subgroup $G_{v_{M}} \subset O\left(q_{N S}\right)$ of $v_{M}$.

Proof. According to the isomorphism in Theorem 4.2 (1), we consider the group

$$
\begin{aligned}
H:=\left\{\left(\alpha_{M}, \alpha_{N}\right) \in O\left(q_{M}\right) \times O\left(q_{N}\right) \mid \alpha_{M}\left(\Gamma_{M}\right)\right. & \\
& \left.=\Gamma_{M}, \alpha_{N}\left(\Gamma_{N}\right)=\Gamma_{N}, \alpha_{M} \gamma=\gamma \alpha_{N}\right\}
\end{aligned}
$$

and decompose $\sigma_{N S}$ as $K \stackrel{p}{\longrightarrow} H \stackrel{q}{\longrightarrow} O\left(q_{N S}\right)$. Since an automorphism in $K$ preserves $\Gamma_{M}$ and $\Gamma_{N}$, it is clear that $\sigma_{N S}(K) \subset G_{v_{M}}$.

We prove the converse, $\sigma_{N S}(K) \supset G_{v_{M}}$. First we note that $p$ is surjective since $\sigma_{M}$ and $\sigma_{N}$ are both surjective as seen in the proof of Proposition 4.3. Thus it is enough to see $\operatorname{im} q \supset G_{v_{M}}$. By Theorem 4.2, we have an isomorphism

$$
q_{M} \cong\left(q_{N} \oplus q_{N S} \mid \Gamma^{\prime \perp}\right) / \Gamma^{\prime}
$$

where $\Gamma^{\prime}$ is an isotropic subgroup of $q_{N} \oplus q_{N S}$ which is a pushout of an isomorphism $\gamma^{\prime}: \Gamma_{N}^{\prime} \rightarrow \Gamma_{N S}^{\prime}$ between subgroups of $A_{N}$ and $A_{N S}$ respectively. Using the same notation of Proposition 4.3, it is easy to see that $\Gamma^{\prime}=\left\{0,\left(z_{N}, v_{M}\right)\right\}$.

Suppose we are given an element $\beta \in G_{v_{M}}$. Then the automorphism

$$
\left(\operatorname{id}_{N}, \beta\right) \in O\left(q_{N}\right) \times O\left(q_{N S}\right)
$$

clearly preserves $\Gamma^{\prime}$ and induces an element $\alpha_{M} \in O\left(q_{M}\right)$ under the isomorphism (1). Since the element $\left(0, v_{M}\right)$ of the right-hand-side of (1) corresponds 
to $z_{M}, \alpha_{M}$ preserves $z_{M}$. By construction $\alpha_{M} \gamma=\gamma \mathrm{id}_{N}$ holds and therefore $\left(\alpha_{M}, \operatorname{id}_{N}\right) \in H$.

Proposition 4.7. Let $M_{1}$ and $M_{2}$ be the fixed lattices of two free involutions $i_{M_{1}}, i_{M_{2}}$ on $X$. Then they give the isomorphic quotients if and only if their patching elements coincide.

Proof. Suppose $X / i_{M_{1}} \cong X / i_{M_{2}}$. There exists $\varphi \in \operatorname{Aut}(X)$ such that $\varphi\left(M_{1}\right)=M_{2} . \varphi$ preserves the overlattice $N S$ so that we have $\varphi\left(z_{M_{1}}\right)=z_{M_{2}}$ and $\sigma_{N S}(\varphi)\left(v_{M_{1}}\right)=v_{M_{2}}$. On the other hand, $\varphi$ acts on $A_{N S}$ trivially by the assumption of the theorem. Thus we see $v_{M_{1}}=v_{M_{2}}$.

Conversely assume the patching elements $v_{M} \in A_{N S}$ concide. By Proposition 4.3, the primitive embeddings $M_{1}$ and $M_{2}$ are isomorphic and there exists $\varphi \in O(N S)$ such that $\varphi\left(M_{1}\right)=M_{2}$, namely $\varphi i_{M_{1}} \varphi^{-1}=i_{M_{2}}$. By assumption, $\sigma_{N S}(\varphi)\left(v_{M}\right)=v_{M}$. We can assume $\varphi \in O^{\uparrow}(N S)$ by replacing $\varphi$ by $-\varphi$ if necessary. According to Proposition $1.3(2), \varphi=w \psi$ where $w \in W_{X}, \psi \in O^{+}(N S)$. Then Lemma $1.4(2)$ implies $\psi\left(M_{1}\right)=\left(M_{2}\right)$. On the other hand, since $w$ acts on $A_{N S}$ trivially, $\sigma_{N S}(\psi)=\sigma_{N S}(\varphi)$ and this element fixes $v_{M}$. We apply Theorem 2.3, Step 3 to $M=M_{1}, \psi_{1}=\psi, \psi_{2}=\operatorname{id}_{N S}$. Both $\psi_{1}$ and id stabilize $v_{M}$, therefore their images by $\sigma_{N S}$ have the same class in $O\left(q_{N S}\right) / \sigma_{N S}(K)$ by the previous lemma. Thus the conclusion holds.

Next we compute the patching elements of involutions of Lieberman and Kondo-Mukai. They involve parameters as mentioned in the beginning of this section and we have to consider the dependence of patching elements on the parameters. This is directly done. We take the following basis of $A_{N S}$. $e_{1}=\left(G_{11}+G_{13}+G_{31}+G_{33}\right) / 2, \quad f_{1}=\left(G_{22}+G_{23}+G_{32}+G_{33}\right) / 2$, $e_{2}=\left(G_{21}+G_{23}+G_{31}+G_{33}\right) / 2, \quad f_{2}=\left(G_{12}+G_{13}+G_{32}+G_{33}\right) / 2$.

Then the result is as in Figures 3 and 4 .

From these figures we can see which patching element we obtain when we choose a parameter of a geometrically constructed free involution. In Figure 4, we normalized the cases to only $i_{4}=j_{4}=4$.

Since the vectors in Figures 3 and 4 run all over $A_{N S}-\{0\}$, we obtain

Proposition 4.8. The two kinds of free involutions gives the all distinct Enriques quotients of $X$.

The proof of Theorem 4.1 is completed.

Remark 4.9. (1) The involution $\tau$ acts trivially on $N S(X)$. So it induces a numerically trivial involution on the fifteen Enriques quotients. The KondoMukai case of this is the last and missing result of [5], first found in [3]. 


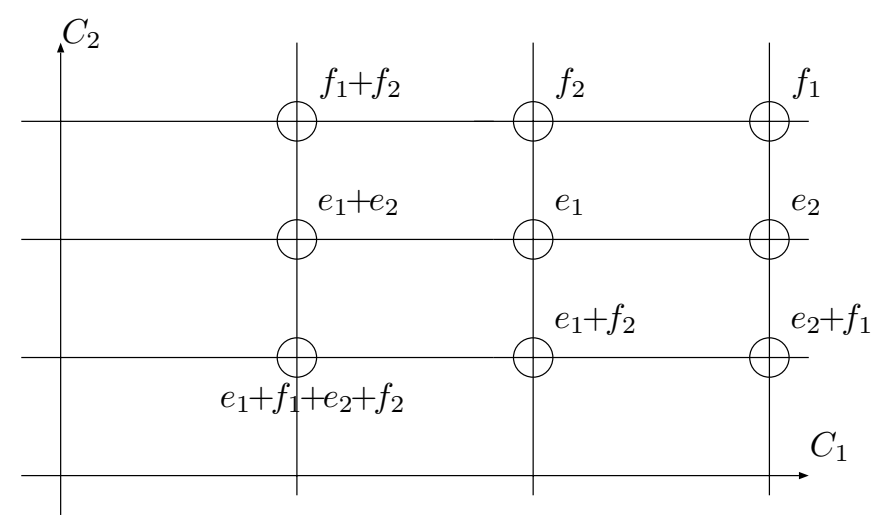

Figure 3. Lieberman involutions

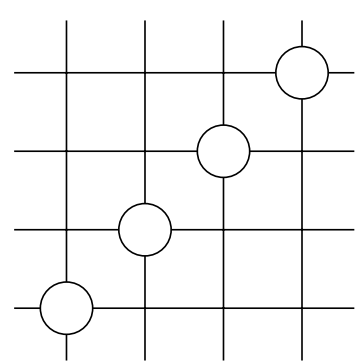

$e_{2}+f_{2}$

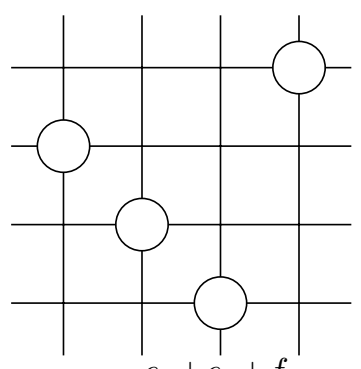

$e_{1}+e_{2}+f_{2}$

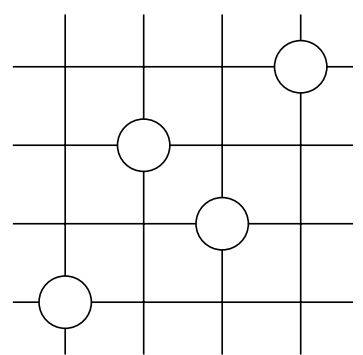

$f_{1}+e_{2}+f_{2}$

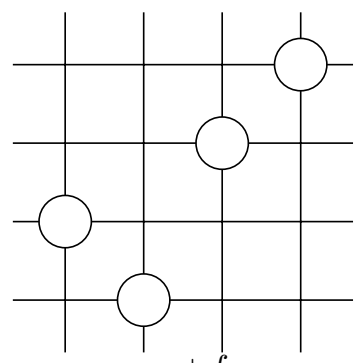

$e_{1}+f_{1}$

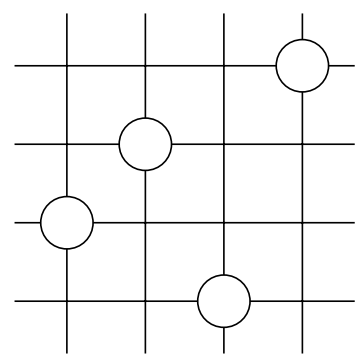

$e_{1}+f_{1}+e_{2}$

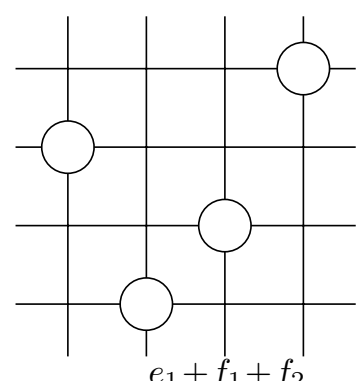

$e_{1}+f_{1}+f_{2}$

Figure 4. Kondo-Mukai involutions 
(2) The number of Enriques quotients can be computed in other 2-elementary cases, using the argument of this section.

When $N S(X) \cong U(2) \oplus E_{8}^{\oplus 2}$, the Barth-Peters case, the number $B_{0}$ is equal to 1 .

When $N S(X) \cong U(2) \oplus E_{8}(2)$, then $X$ has only one Enriques quotient. Finally using the result of [5], we see that in other 2-elementary cases $X$ has no Enriques quotients.

(3) The generators of the whole automorphism group $\operatorname{Aut}(X)$ are found in [2].

\section{References}

[1] W. Barth and C. Peters, Automorphisms of Enriques surfaces, Invent. Math. 73 (1983), no. 3, 383-411.

[2] J. Keum and S. Kondō, The automorphism groups of Kummer surfaces associated with the product of two elliptic curves, Trans. Amer. Math. Soc. 353 (2001), no. 4, 1469-1487 (electronic).

[3] S. Kondō, Enriques surfaces with finite automorphism groups, Japan. J. Math. (N.S.) 12 (1986), no. 2, 191-282.

[4] S. Mukai, Numerically trivial involutions of an Enriques surface. (preprint, 2006)

[5] S. Mukai and Y. Namikawa, Automorphisms of Enriques surfaces which act trivially on the cohomology groups, Invent. Math. 77 (1984), no. 3, 383-397.

[6] Y. Namikawa, Periods of Enriques surfaces, Math. Ann. 270 (1985), no. 2, 201-222.

[7] V. V. Nikulin, Integral symmetric bilinear forms and some of their applications (English translation). Math. USSR Izv. 14 (1980), 103-167.

[8] Finite automorphism groups of Kähler $K 3$ surfaces (English translation). Trans. Moscow Math. Soc. Issue 2 (1980), 75-137.

[9] subgroups generated by 2-reflections (English translation). J. Soviet Math. 22 (1983), 1401-1476.

[10] I. Z. Pjatečkili-S̆apiro and I. R. S̆afarevič , A Torelli theorem for algebraic surfaces of type K3 (English translation). Math. USSR Izv. 5 (1971), 547-588.

[11] V. Platonov and A. Rapinchuk, Algebraic groups and number theory, Translated from the 1991 Russian original by Rachel Rowen, Academic Press, Boston, MA, 1994.

[12] È. B. Vinberg, Some arithmetical discrete groups in Lobačevskiǔ spaces, in Discrete subgroups of Lie groups and applications to moduli (Internat. Colloq., Bombay, 1973), 323-348, Oxford Univ. Press, Bombay. 